\title{
Waves on the dusk flank boundary layer during very northward interplanetary magnetic field conditions: Observations and simulation
}

\author{
D. H. Fairfield, ${ }^{1}$ M. M. Kuznetsova, ${ }^{1}$ T. Mukai, ${ }^{2}$ T. Nagai, ${ }^{3}$ T. I. Gombosi, ${ }^{4}$ \\ and A. J. Ridley ${ }^{4}$ \\ Received 8 September 2006; revised 21 February 2007; accepted 2 May 2007; published 9 August 2007.
}

[1] The Geotail spacecraft made an inbound passage perpendicular to the dusk equatorial magnetopause on 1 August 1998 when the interplanetary magnetic field had been very northward for more than 10 hours. As the spacecraft moved through the low-latitude boundary layer, it detected waves with $\sim 3$ min period that caused transitions between cool dense magnetosheath plasma and mixed magnetosheath and magnetosphere plasmas. A region of highly fluctuating magnetic fields with variations up to $50 \mathrm{nT} / \mathrm{s}$ was observed on leaving the magnetosheath plasma but not on its return to this region. This observation suggests that the boundary region is not laminar but more likely consists of KelvinHelmholtz vortices. Such fluctuations are probably caused by twisting of the field by the vortices, and they could be important in transporting plasma to the magnetosphere where densities of 5/cc were observed. A global MHD simulation of the event reproduced the observed low-frequency waves, their counterclockwise polarization, and a region of high-velocity magnetosheath flow outside the magnetopause. This flow was associated with a decreased density and increased field strength which are characteristics of a plasma depletion layer. When Geotail moved further inward into the magnetosphere, it detected waves that are probably the magnetotail vortices which had been detected earlier by the ISEE spacecraft.

Citation: Fairfield, D. H., M. M. Kuznetsova, T. Mukai, T. Nagai, T. I. Gombosi, and A. J. Ridley (2007), Waves on the dusk flank boundary layer during very northward interplanetary magnetic field conditions: Observations and simulation, J. Geophys. Res., 112, A08206, doi:10.1029/2006JA012052.

\section{Introduction}

[2] Waves on flanks of the magnetosphere have been reported by many observers [e.g., Ogilvie and Fitzenreiter, 1989; Chen et al., 1993; Chen and Kivelson, 1993; Kivelson and Chen, 1995; Takahashi et al., 1991; Kokubun et al., 1994; Seon et al., 1995; Fairfield et al., 2000, 2003; Farrugia et al., 2000; Owen et al., 2004; Hasegawa et al., 2004]. These waves have frequently been attributed to the Kelvin-Helmholtz $(\mathrm{KH})$ instability caused by shear between the high-velocity magnetosheath plasma and the nearly stagnant magnetosphere plasma. The waves are important because they are thought to cause momentum, energy, and perhaps mass transfer into the magnetosphere. They also may be a source of Pc 5 magnetospheric waves that are believed to energize trapped electrons [e.g., O'Brien

\footnotetext{
${ }^{1}$ Laboratory for Solar and Space Physics, NASA Goddard Space Flight Center, Greenbelt, Maryland, USA.

${ }^{2}$ Institute of Space and Astronautical Science, Sagamihara, Kanagawa, Japan.

${ }^{3}$ Earth and Planetary Sciences, Tokyo Institute of Technology, Tokyo, Japan.

${ }^{4}$ Center for Space Environment Modeling, University of Michigan, Ann Arbor, Michigan, USA.

Copyright 2007 by the American Geophysical Union. 0148-0227/07/2006JA012052
}

and McPherron, 2003; Li and Temerin, 2001; Mann et al., 2002].

[3] Many papers report on simulations of the KH instability. The majority of these simulations use two-dimensional compressible MHD codes, usually with periodic boundary conditions [Miura, 1984, 1987, 1990, 1995, 2003; Keller and Lysak, 1999; Otto and Fairfield, 2000; Nykyri and Otto, 2001; Matsumoto and Hoshino, 2004, 2006]. Such simulations demonstrate the time development of one wavelength; however, they fail to place the phenomena in a global context. Initial conditions are usually chosen to represent the dayside magnetopause where magnetosheath $\mathrm{B}$ is usually less than magnetosphere B. Reported simulations achieve the $\mathrm{KH}$ vortices they set out to study, but typically little is said about whether growth rates are large enough to grow significant magnetopause waves before the waves would move downstream of an observing point in an actual magnetosphere. Wright et al. [2000, 2002] and Mills et al. [2000] refer to a body of plasma physics literature and discuss this important propagation question in terms of a wave packet that is "absolutely" unstable (it grows fast enough to affect its point of origin) or "convectively" unstable (it propagates away fast enough that it decays at its point of origin). Given a large enough growth rate, the instability will be absolutely unstable and vortices will develop where magnetosheath plasma becomes entrained in a spiraling vortex. These vortices become increasingly 
complex with time as the instability approaches saturation [e.g., Thomas and Winske, 1993; Matsumoto and Hoshino, 2004].

[4] Reconnection has been suggested as a process occurring within a vortex [Otto and Fairfield, 2000; Nykyri and Otto, 2001]. Nakamura et al. [2004, 2006] and Nakamura and Fujimoto [2005] pursue this idea using a two-fluid model including electron inertial effects. They demonstrate the importance of reconnection within a rolled up vortex in allowing plasma entry into the magnetosphere, a transfer that would not occur in ideal MHD. Matsumoto and Hoshino [2004] using two-dimensional MHD simulations emphasize the importance of a density change across the boundary which can lead to secondary $\mathrm{KH}$ and RayleighTaylor instabilities within a vortex leading to turbulence and mixing of the magnetosheath and magnetosphere plasmas. Here the centrifugal force of the initial rotating $\mathrm{KH}$ vortex plays the role of gravity in the Rayleigh-Taylor instability. Lai and Lyu [2006] emphasize the nonlinear evolution of the $\mathrm{KH}$ instability and its dependence on various parameters. Many simulations make it clear that the waves develop faster when there is a weaker stabilizing magnetosheath field component parallel to the velocity and transverse to the magnetosphere field. [e.g., Miura, 1995; Keller and Lysak, 1999]. This is apparently the reason that most reported observations occur during periods of northward IMF when magnetosheath fields will tend to be perpendicular to the flow and aligned with equatorial magnetosphere fields.

[5] Several hybrid [Thomas and Winske, 1993; Thomas, 1995; Fujimoto and Terasawa, 1994, 1995] and particle [Matsumoto and Hoshino, 2006] KH simulations have been reported, again with periodic boundary conditions. These studies find results similar to those of MHD but often with structures breaking off of irregular vortices [e.g., Thomas and Winske, 1993]. The focus of these studies is on mixing of magnetosphere and magnetosheath plasmas. Maximum mixing is often found in the simple, but unrealistic, case of uniform density and field across the velocity shear. Thomas and Winske [1993] find that a density change across the boundary suppresses vortex development and mixing, whereas Matsumoto and Hoshino [2006] find larger density changes leads to secondary instabilities and increased mixing. Fujimoto and Terasawa [1995] find that a B magnitude change across the boundary decreases mixing. The Thomas [1995] paper includes a relatively simple three-dimensional (3-D) hybrid simulation with uniform density and B, and he finds the 3-D results generally correspond rather well to 2-D. When B rotates across the shear layer, however, the instability is strongly inhibited and Thomas questions whether $\mathrm{KH}$ is a viable mechanism on the dayside magnetosphere except under the rare, very northward IMF conditions that align the fields across the boundary. Takagi et al. [2006] use a 3-D MHD simulation with northward magnetosheath field to investigate a situation where an unstable plasma sheet is sandwiched between stable tail lobes. They find that the plasma sheet instability persists and forms a highly rolled up vortex even when the plasma sheet thickness is smaller than the wavelength of the fastest growing mode. Knoll and Brackbill [2002] also perform a 3-D MHD simulation with $\mathbf{B}$ perpendicular to $\mathbf{V}$; they emphasize the reconnection that results from field twisting due to differential rotation along the $\mathbf{B}$ direction.
[6] Manuel and Samson [1993] use a MHD code and nonperiodic boundary conditions that allow them to investigate a series of vortices and show how the earlierdeveloping vortices act back on the later developing ones. In running several cases, they demonstrate how the field component in the flow direction inhibits the growth and the size of the vortices. They can also see how adjacent vortices can merge. Slinker et al. [2003] simulate a specific Geotail event with a global MHD code. While they cannot fully resolve the boundary layer, they find evidence for vortices and wave frequencies and amplitudes that agrees rather well with observations.

[7] These simulations of the KH instability reveal useful details of how the instability develops through linear and nonlinear stages until saturation. Whether the instability develops and how rapidly it progresses through these stages is highly dependent on prevailing conditions. Trying to relate spacecraft measurements to a theoretical boundary layer is difficult, particularly since it is never completely clear where the spacecraft is located relative to the presumed spatial structure. Below we will investigate a case on 1 August 1998 when the Geotail spacecraft made an inbound pass nearly perpendicular to the dusk boundary layer when the solar wind was relatively steady and the IMF was almost directly northward, thus replicating conditions when the boundary layer is most apt to be KH unstable. Large-scale boundary motions due to solar wind changes were thus minimized and the spatial structure was better able to be evaluated as the spacecraft moved steadily inward. We compare the observations to a global simulation of the event which, though unable to replicate the complex details of small-scale structure, shows evidence of $\mathrm{KH}$ waves and reveals the spatial variations of the magnetosheath parameters that control the instability. The simulation also allows study of the magnetosphere waves generated by the instability and seen in the observations.

\section{Spacecraft Position and Interplanetary Conditions}

[8] The trajectory of Geotail for the hours of 0000-1800 1 August 1998 is shown in Figure 1 along with the Shue model magnetopause calculated with the observed solar wind pressure and $\mathrm{B}_{\mathrm{z}}$ of $2.5 \mathrm{nPa}$ and $9 \mathrm{nT}$. The interval of boundary layer observation at dusk, 0530-0730, is marked on the trajectory with a heavy line. Geotail is close to the equator at 0530; the GSM Z position is $\sim-3 \mathrm{R}_{\mathrm{E}}$ but a sunward tilt of the dipole of $\sim 8^{\circ}$ raises the equatorial current sheet near midnight but lowers this effective equatorial plane on the flanks by about $2 \mathrm{R}_{\mathrm{E}}$ according to the a neutral sheet model [Tsyganenko and Fairfield, 2004] so that the spacecraft is expected to be about $1 \mathrm{R}_{\mathrm{E}}$ south of the actual equator. Small observed negative $B_{x}$ and positive $B_{y}$ components confirm this southern location.

[9] Interplanetary conditions measured by the Wind spacecraft (the Solar Wind Plasma Experiment (SWE) [Ogilvie et al., 1995] and the Magnetic Field Investigation (MFI) [Lepping et al., 1995]) at a solar ecliptic location $\left(\mathrm{X}=87, \mathrm{Y}=-7, \mathrm{Z}=-3 \mathrm{R}_{\mathrm{E}}\right)$ are displayed in Figure 2. The clock angle, CA, is defined such that $0^{\circ}$ is northward, plus or minus $180^{\circ}$ is southward, and plus and minus $90^{\circ}$ are equatorial in the positive and negative $\mathrm{Y}$ direction, 


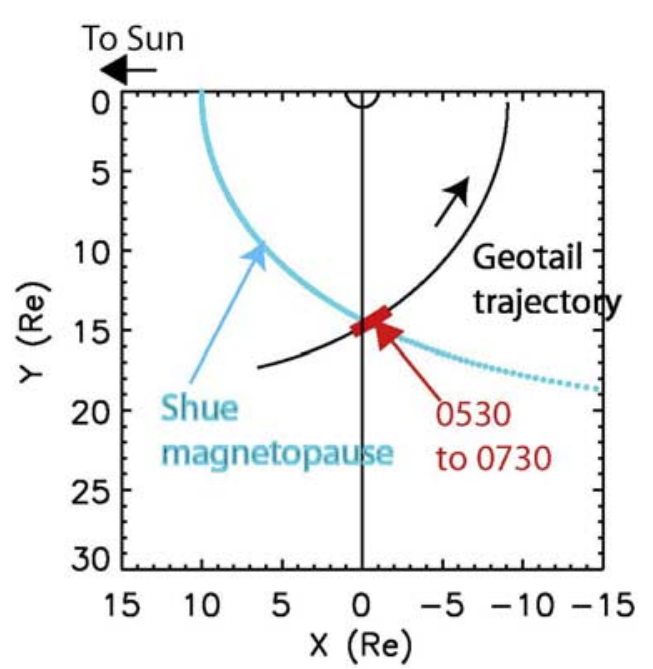

Figure 1. Inbound trajectory of Geotail near the dusk terminator on 1 August 1998. The heavy line segment as Geotail crosses the magnetopause designates the 05300730 interval of particular interest.

respectively. The data are shifted $23 \mathrm{~min}$ to make them correspond to their Earth arrival time. Data are in solar magnetospheric coordinates and the interval of the Geotail boundary crossing is spanned by vertical dashed lines. The preceding interval is shown to emphasize that Geotail measurements were made during an unusually long interval of northward IMF $\left(\sim 0^{\circ}\right.$ clock angle).

\section{Geotail Observations}

[10] Figure 3 presents an overview of the Geotail data during the low-latitude boundary layer crossing interval. Figure 3 a repeats the solar wind kinetic pressure of Figure 2 shifted 23 min to its Earth arrival time. The red traces in Figures $3 \mathrm{~b}, 3 \mathrm{c}, 3 \mathrm{~d}, 3 \mathrm{f}, 3 \mathrm{~g}$, and $3 \mathrm{i}$ correspond to plasma density, temperature, velocity, solar magnetospheric flow direction angles $\theta$ and $\phi$, and plasma pressure, all measured by the Geotail LEP plasma experiment [Mukai et al., 1994] and shown at $12 \mathrm{~s}$ resolution. This experiment has separate instruments to measure the solar wind and the hot plasmas of the magnetosphere. Most measurements after 0545 are made by the hot plasma analyzer and can be taken with some confidence. At earlier times when the ion fluxes are large, however, this instrument switches to a protective mode where only the higher energies are measured. Such cases usually correspond to rapidly flowing magnetosheath plasma, so data from the solar wind instrument is substituted in the data set. At the Geotail location near the dusk terminator, however, this sensitive solar wind detector becomes saturated and the magnetosheath flow is also deflected partly out the viewing range of this instrument (the solar direction $\pm 45^{\circ}$ ). For these reasons, LEP densities, while useful, are not accurate in this high flux region. This suspect LEP data from the solar wind instrument is plotted with open squares and not connected with lines in Figures $3 b$, $3 \mathrm{c}$, and $3 \mathrm{~d}$. To supplement the LEP data, density from the Geotail Comprehensive Plasma Instrument (CPI) [Frank et al., 1994], with lower time resolution and lower sensitivity but greater accuracy, is plotted in blue in the high flux region. The magnetic field magnitude and solar magnetospheric latitude and longitude angles $\theta$ and $\phi$ are shown in Figures $3 \mathrm{e}, 3 \mathrm{f}$, and $3 \mathrm{~g}$ at $3 \mathrm{~s}$ resolution. The RMS values over the $3 \mathrm{~s}$ interval are shown in Figure 3j. Plasma proton beta is shown in Figure $3 \mathrm{~h}$ and the separate magnetic field and plasma pressures are shown by the black and red traces, respectively, in Figure 3i with their sum in green.

[11] In the overview in Figure 3, Geotail moved from the dense magnetosheath to the lower density boundary layer at 0530 UT as marked by the vertical dashed line. This boundary layer has an unusually high density of $\sim 5 / \mathrm{cc}$ and it is most easily identified by the higher ion temperatures and variable field magnitudes and densities, all of which oscillate as Geotail periodically detects magnetosheath-like plasma. Neither the field or flow direction angles change at the magnetopause, indicating that the boundary layer and magnetosheath fields are closely aligned and the outer portion of the boundary layer is flowing tailward, although with lower velocity than the magnetosheath. A more subtle change in the boundary layer occurs

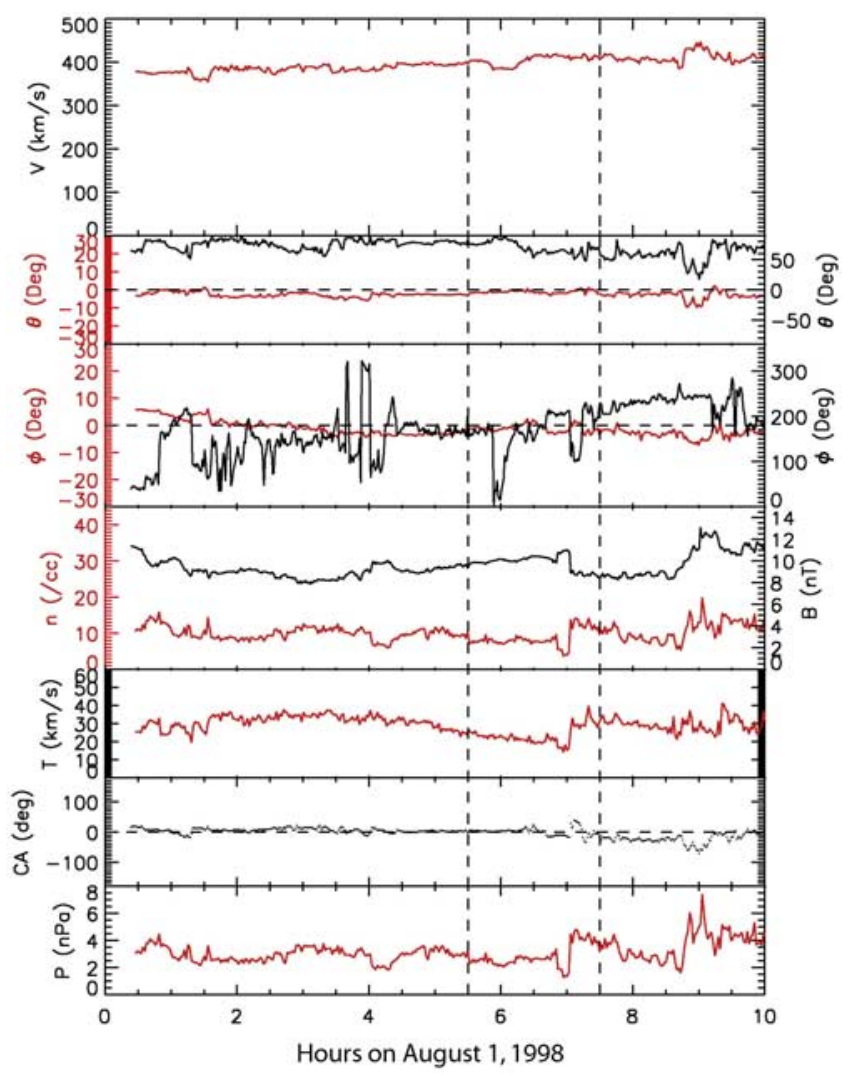

Figure 2. Solar wind (red) and magnetic field (black) parameters measured by the Wind spacecraft are shown around the time of the Geotail boundary crossing interval designated by vertical dashed lines. Shown are velocity V, solar magnetospheric latitude and longitude angles $\theta$ and $\phi$, density $n$, magnetic field magnitude $B$, ion temperature $T$, clock angle $\mathrm{CA}$, and kinetic pressure $\mathrm{P}$. The magnetic field latitude angle $\theta$ is very northward for many hours preceding and including the interval of interest. $\mathrm{P}$ is relatively steady throughout the interval of interest excepting near 0700 . 


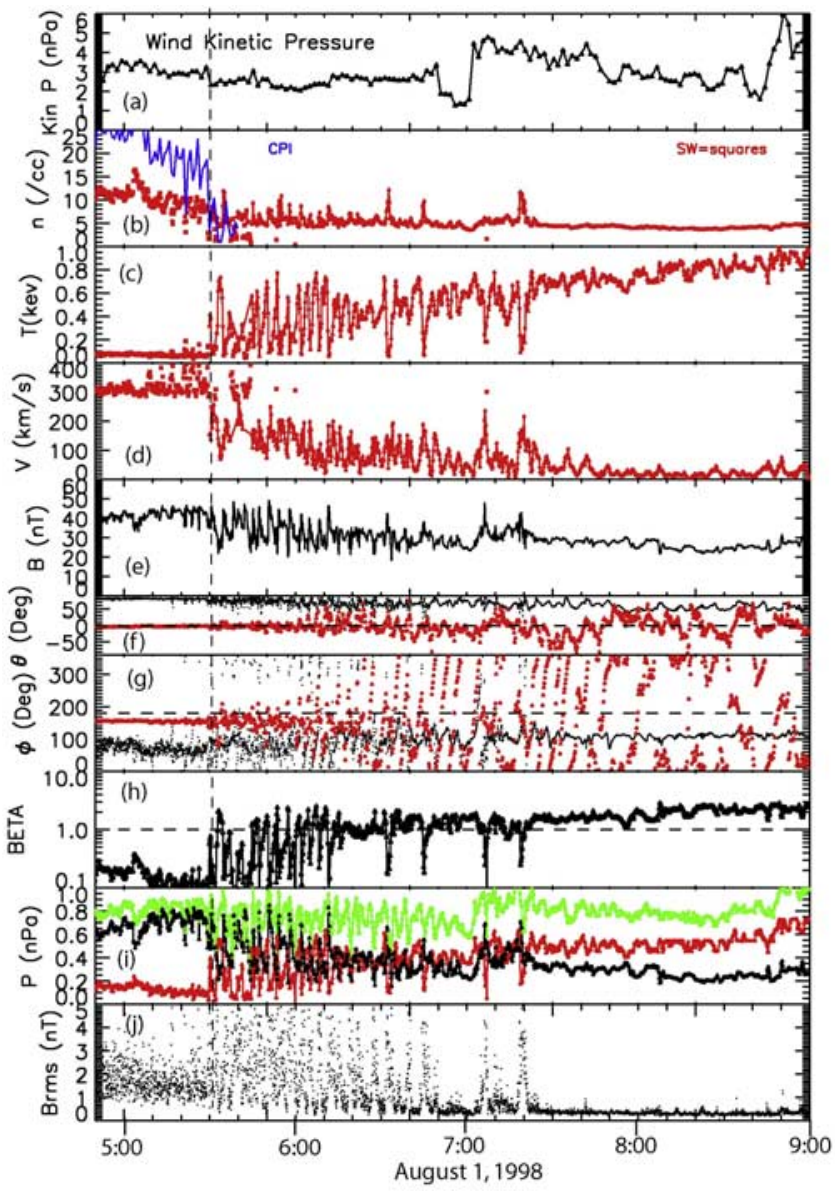

Figure 3. An overview of Geotail magnetic field and plasma parameters during the boundary layer crossing interval. The magnetopause is designated with a vertical dashed line.

near 0613 when detection of the magnetosheath-like plasma become infrequent as indicated by the lack of higher densities and magnetic field strengths. It may also be significant that at this time the IMF becomes somewhat less northward, causing the clock angle to begin changing from zero to slightly positive values. Also beginning near 0613 UT, flows with a sunward component are occasionally detected by Geotail, as indicated by $\phi$ angles less than $90^{\circ}$ or greater than $270^{\circ}$. As time progresses the $\phi$ flow angles more frequently exhibit gradual increases from $0^{\circ}$ to $360^{\circ}$, indicating a counter clockwise rotation of the flow vector (looking down from the north). The two primary solar wind pressure increases at 0701 and 0845 UT correspond to increases in the total Geotail thermal plus magnetic pressure. After the solar wind pressure increase at $0701 \mathrm{UT}$ and a subsequent decrease, the flow more frequently has a sunward component, although the magnitudes are small. The above characteristics will be shown more clearly in subsequent figures. Note that LEP plasma beta throughout the boundary layer is generally between 1 and 2 compared to a magnetosheath value that is much lower even if correct magnetosheath density had been used.

[12] Figure $4 \mathrm{~b}$ shows the perpendicular distance, D, between Geotail and the Shue et al. [1998] model magne- topause that is calculated as a function of time using the measured kinetic pressure and IMF $B_{z}$ shown in Figures $4 c$ and $4 \mathrm{~d}$. Positive values of $\mathrm{D}$ indicate predicted locations outside the boundary and negative values inside. Figure 4a repeats the Geotail temperature from Figure 3 as an indicator of boundary layer properties. The solar wind pressure decrease at 0530 UT apparently caused expansion of the magnetosphere and the initial magnetopause crossing at 0530 UT. Geotail detected the low temperatures characteristic of magnetosheath plasmas every wave cycle until 0613 UT and then occasionally until 0720 UT. Between 0530 and 0613 UT Geotail moved inward relative to the boundary by almost $1 \mathrm{R}_{\mathrm{E}}$. This distance can be taken as an upper limit to the boundary layer thickness since there is no gradual variation of the controlling parameters during this interval that would move the average boundary location relative to the inward moving spacecraft. The more sudden variations in the various parameters suggest that the actual boundary layer thickness may be less than $1 \mathrm{R}_{\mathrm{E}}$. As the spacecraft moved further inward after 0720 UT, it no longer encountered magnetosheath-like plasma even though it continued to see waves in velocity measurements, as will be shown below.

[13] An expanded view of the interval 0545-0615 UT using high-resolution magnetic field (16 vectors/s) is shown in Figure 5 where most of the parameters of Figure 3 are shown along with vector components. The data are in solar magnetospheric coordinates except that velocities have been rotated by $26^{\circ}$ about the $\mathrm{Z}$ axis into a coordinate system aligned with an average boundary where $\mathrm{Y}$ is perpendicular to the boundary. Again plasma data is in red and magnetic field in black. Vertical dashed blue lines delineate sharp increases in B which correspond to increases in density and decreases in temperature, all of which are characteristic of sudden encounters with magnetosheath plasma. These sudden changes followed by slow recoveries create a sawtooth appearance often reported in previous measurements [e.g., Chen et al., 1993; Fairfield et al., 2000]. A periodicity of

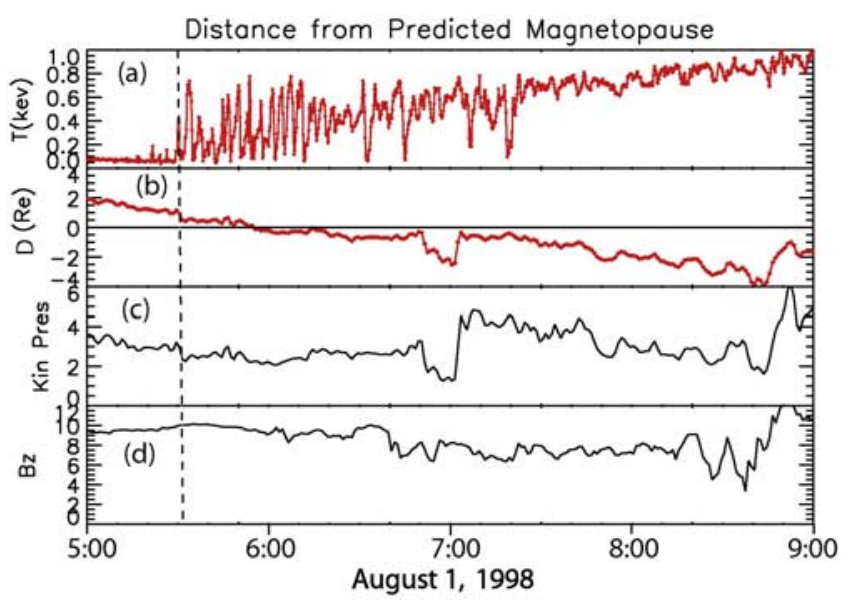

Figure 4. (a) The fluctuating ion temperature, T, identifies the boundary layer that follows the inbound magnetopause crossing which is indicated by the vertical dashed line. Also indicated is (b) the distance from the Shue model magnetopause calculated with (c) the solar wind pressure and (d) $B_{z}$. 


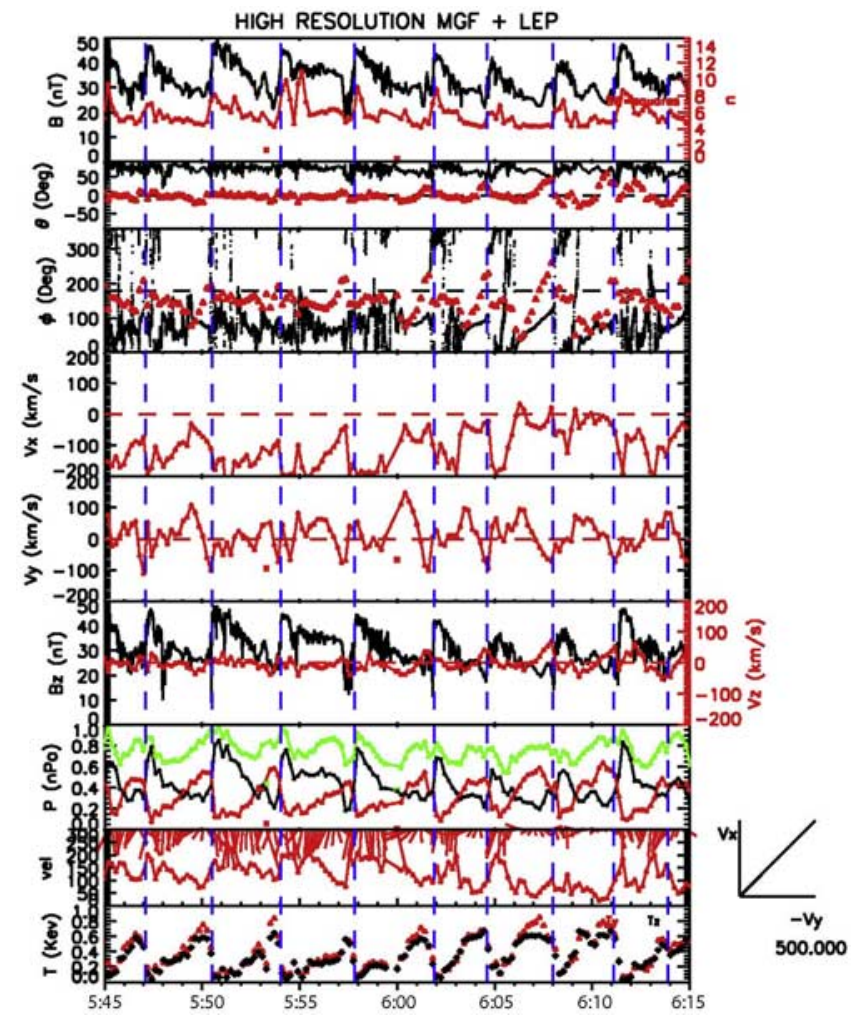

Figure 5. An expanded interval of Figure 3 showing most of the same parameters but adding vector components. XY flow vectors are shown in the velocity panel with tailward downward and duskward to the left. Vxy vectors are defined by the axes schematic in the bottom right of the figure. Ion temperature along $\left(\mathrm{T}_{\mathrm{z}}\right)$ and perpendicular to $\left(\mathrm{T}_{\mathrm{y}}\right)$ the spacecraft spin axis is shown in red and black at the bottom.

approximately $3 \mathrm{~min}$ is readily apparent. Very large magnetic field fluctuations are present as B is decreasing but they disappear before the sudden next $\mathrm{B}$ increase. These fluctuations can be discerned in $\mathrm{B}_{\mathrm{z}}$ even though the effect is more prominent in $\mathrm{B}_{\mathrm{x}}$ and $\mathrm{B}_{\mathrm{y}}$ (not shown to prevent crowding). During each wave cycle the regions of highest temperature correspond to low $\mathrm{V}_{\mathrm{x}} . \mathrm{V}_{\mathrm{y}}$ changes from positive to negative toward the end of each cycle and usually reaches a negative peak at the time of the sudden B change. Such a periodicity suggests a tailward moving wave that moves an inner, hotter region outward over the spacecraft and then back inward.

[14] If one simply assumes that waves move a laminar boundary consisting of a fluctuating field region sandwiched between the cool high-density, high field magnetosheath and the more tenuous, hot lower B magnetosphere, it is difficult to explain why the fluctuating region is absent before entering the magnetosheath-like plasma but present when leaving it. A preferred explanation is to invoke a structure such as shown in the top portion of Figure 6 which is adapted from a local MHD simulation of the KelvinHelmholtz instability by Matsumoto and Hoshino [2004, 2006]. We can view this figure as the dusk magnetopause with the Sun to the right even though the simulation is carried out in a plasma reference frame moving tailward. The red region is a dense, cool magnetosheath plasma with high $\mathrm{B}$ flowing to the left and the blue as tenuous, hot magnetosphere plasma with weaker B flowing to the right. The two regions become mixed as they are caught up in Kelvin-Helmholtz vortices. The bottom portion of Figure 6 shows $8 \mathrm{~min}$ of Geotail data extracted from the Figure 5 interval which displays two waves cycles. The white line in the simulation illustrates an imagined Geotail trajectory as the structures move tailward. The white numbers on the simulation plot correspond to the green numbers in the

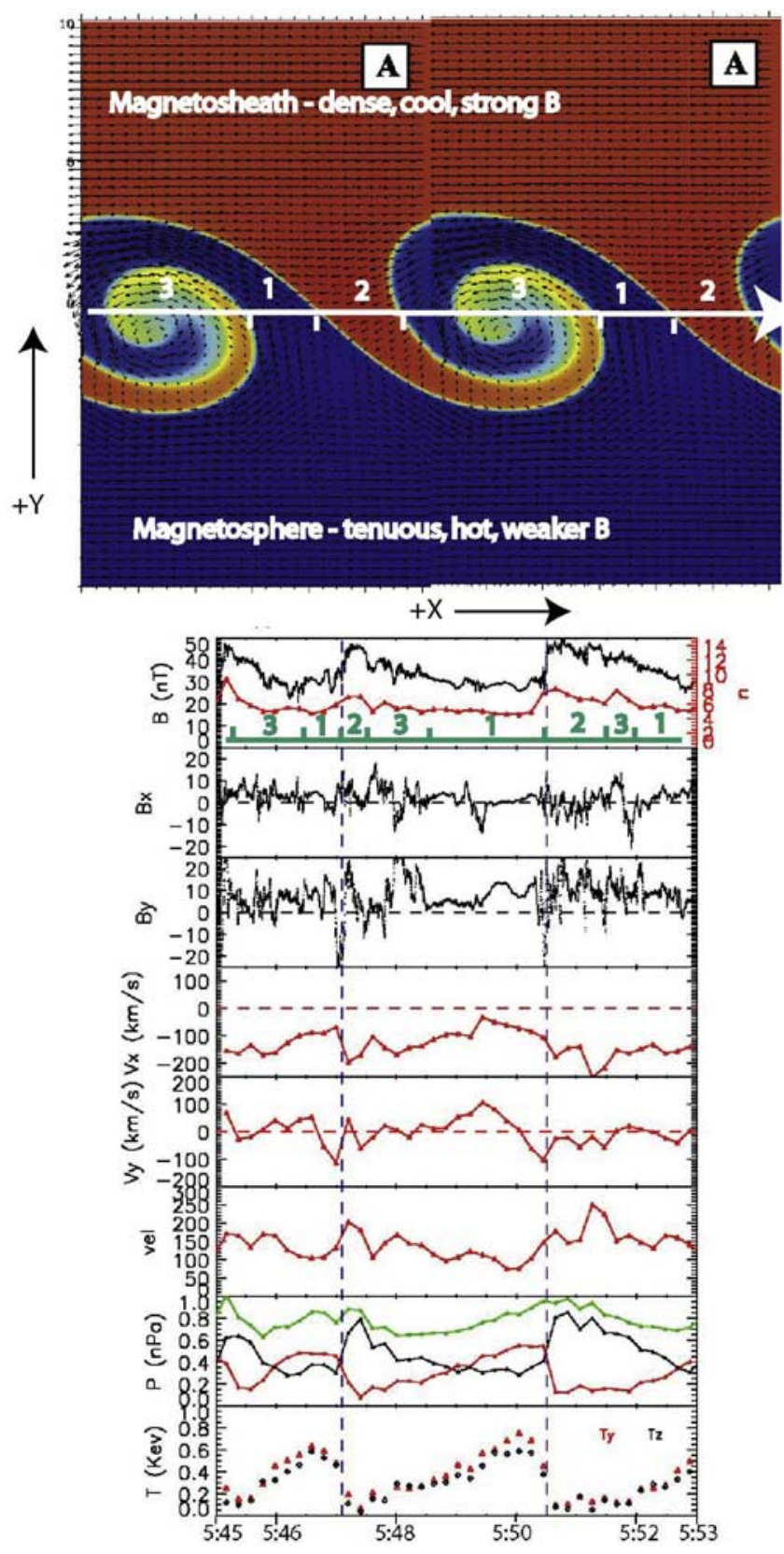

Figure 6. (top) Kelvin-Helmholtz vortices adapted from a figure of Matsumoto and Hoshino [2006], with red colors indicating high densities of the magnetosheath and blue indicating low densities of the magnetosphere. (bottom) Eight minutes of Geotail data are shown, with the numbered regions in the $\mathrm{B}$ panel corresponding to the numbers in the simulation panel (see text). 


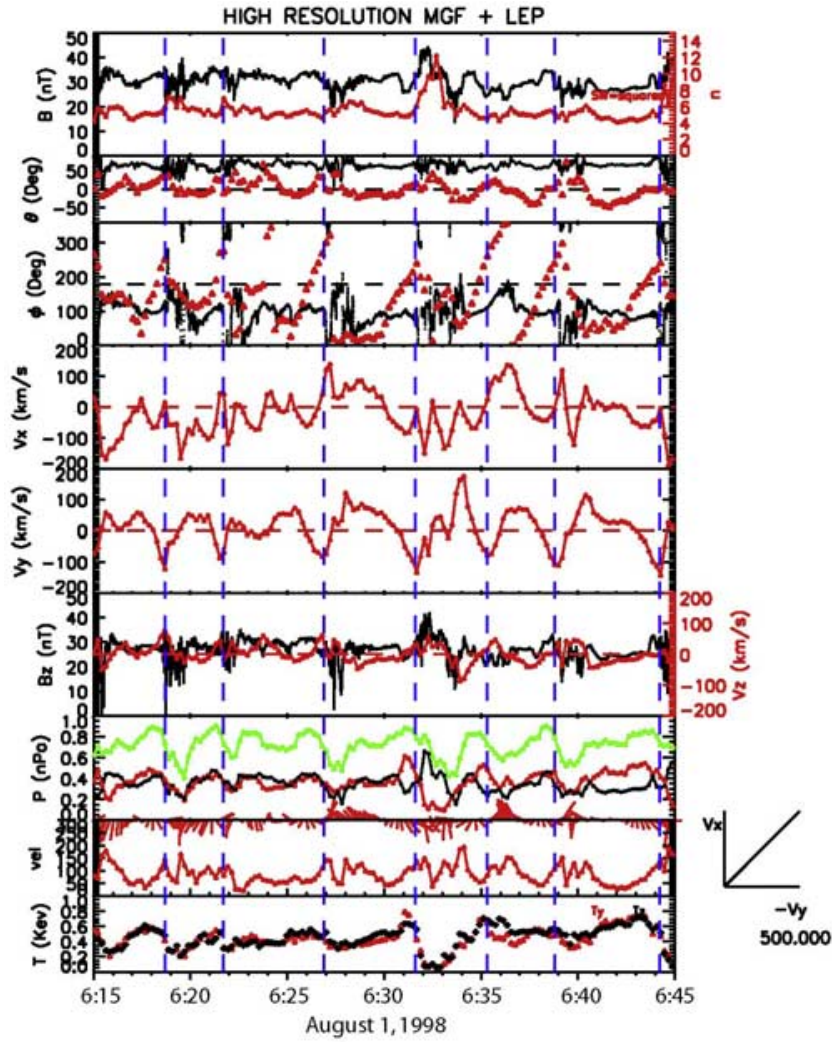

Figure 7. The half hour interval following that of Figure 5. The format is the same. At this more inward location waves persist at a similar frequency as before, but the rapid tailward flows and the characteristics attributed to immersion in vortices are no longer seen.

observed B panel below. The 1 to 2 transition is the sharp boundary between magnetosphere plasmas moving generally upward (positive $\mathrm{Y}$ ) and the magnetosheath plasmas moving generally downward (negative Y). The strongest fields and highest densities usually decrease across the more diffuse 2-3 boundary as the spacecraft moves toward the center of the vortex where the plasmas are mixed. $V_{y}$ velocities tend to change from negative to positive in this region as would be expected on passing through the center of the vortex. The 3 to 1 boundary is characterized by moving from the mixed-plasma, fluctuating-B region to the more purely tenuous, hot magnetosphere plasma region with positive $\mathrm{V}_{\mathrm{y}}$. Throughout this region the plasma and field pressures anticorrelate which tends to keep the pressure constant. Despite this tendency, Region 3 corresponds to a minimum in the total pressure as might be expected in the center of a vortex where centrifugal forces cause the plasma to move outward.

[15] A change in character of the data occurred at about 0615 UT such that the periodic peaks in B and $n$ with low T are no longer present, as can be seen in the data of Figure 7 for 0615 to $0645 \mathrm{UT}$. In the absence of B increases the vertical dashed lines are drawn at negative $V_{y}$ peaks which tended to correspond to the B increases when they occurred in the earlier data. The intervals of high fluctuation still periodically recur but now the associated higher densities and lower temperatures are more modest, except near 0633 UT.
The rapid tailward flows are also absent. The higher temperatures and lower velocities suggest that Geotail is spending more time in a region further in from the boundary, and indeed Figure $4 \mathrm{~b}$ shows that Geotail has moved inward by more than $0.5 \mathrm{R}_{\mathrm{E}}$. Also negative $\mathrm{V}_{\mathrm{y}}$ corresponds to transitions from the hotter, quiet field inner region to the cooler fluctuating region as if an entire vortex was moving inward over the spacecraft. Positive $\mathrm{V}_{\mathrm{y}}$ corresponds to the reverse. Velocity vectors tend to rotate in a counterclockwise manner, as can be seen from the steadily increasing $\phi$ angle in the third panel. The typical wave period is now closer to $4 \mathrm{~min}$. In contrast to the previous half hour, the field and plasma pressures more often increase and decrease together leading to large variations in total pressure. The exception is near 0633 UT where the data are more like the previous half hour. The reports of $\mathrm{KH}$ wave observations cited earlier invariably showed examples where the spacecraft remained in the fluctuation layer and hence did not see the waves in the interior region shown here.

[16] In the next half hour shown in Figure 8, data from 0645-0715 UT again show the periodicity in the counterclockwise rotating flow vector. The interval 0648-0700 UT corresponds to an interval of low solar wind pressure that would expand the magnetopause and move Geotail deeper into the magnetosphere. Indeed no fluctuating boundary layer fields are seen during this interval although they are seen before and after this interval of low solar wind pressure. In this more interior region, plasma and field

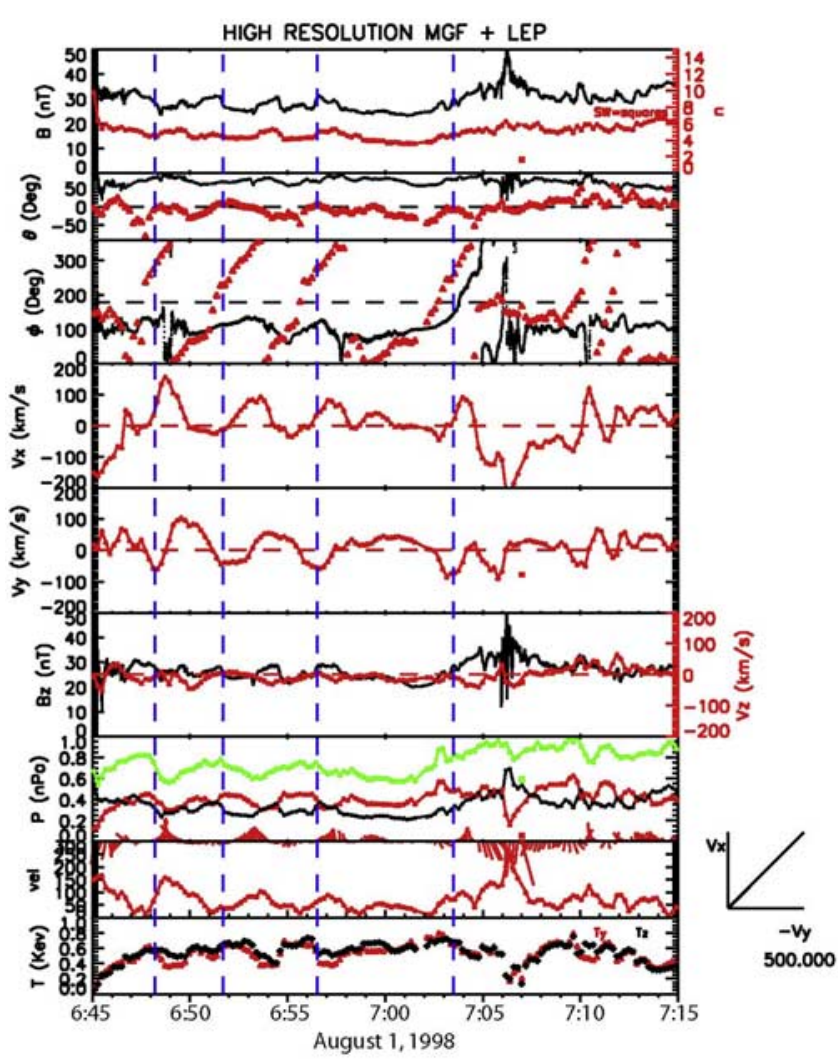

Figure 8. The half hour interval following that of Figure 7 and in the same format. A solar wind pressure increase at 0700 causes increased magnetosphere pressure and the brief reappearance of the fluctuating field region at 0706 . 


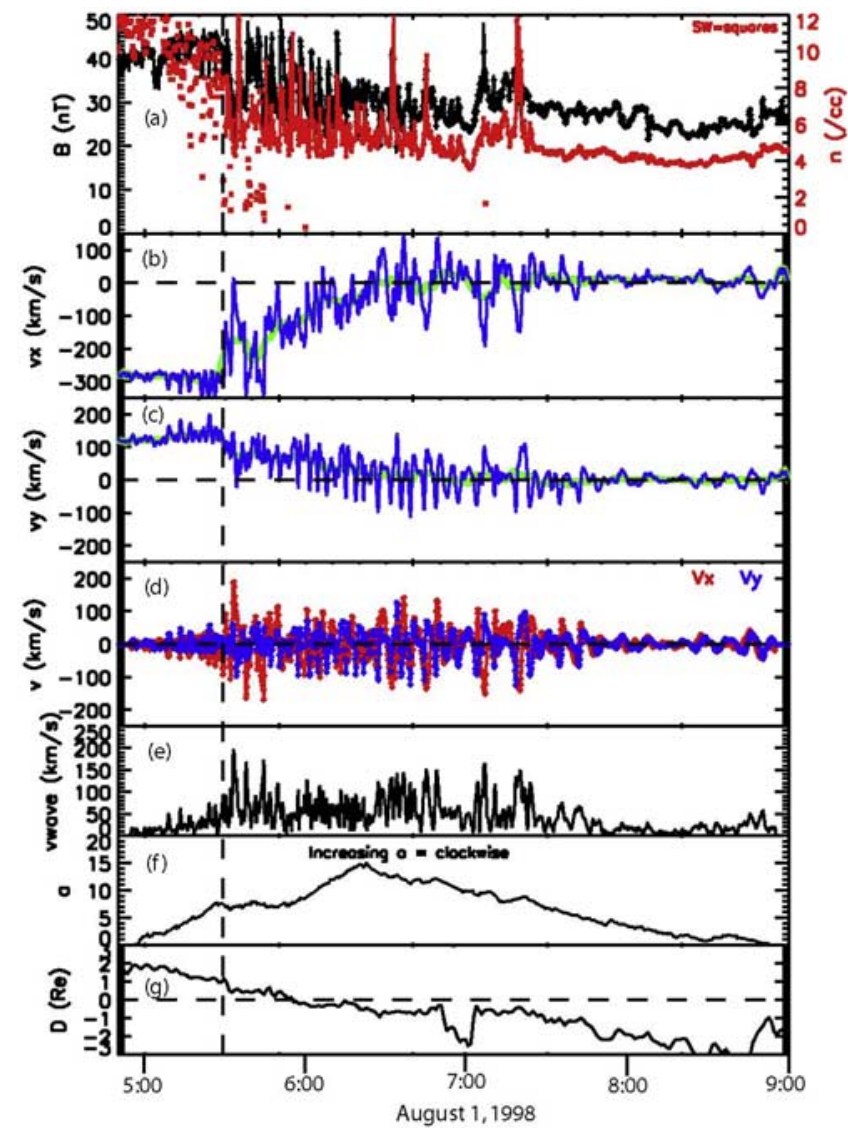

Figure 9. $(a-g)$ The parameter " $a$ " in Figure 9f (see text) as a measure of the velocity vector rotation shows counterclockwise rotation of the magnetosphere plasma in the region $2 \mathrm{Re}$ earthward of the magnetopause.

pressure tend to anticorrelate and keep the total pressure more constant than earlier.

[17] One final brief entry into the high-density and velocity outer boundary layer occurs at 0720 as can be seen in Figure 3. After this time velocities decrease and after 0800, when the spacecraft has moved more than $2 R_{E}$ inside the magnetopause (solar magnetospheric position -2.2, $12.8,-3.7 \mathrm{R}_{\mathrm{E}}$ ), the velocity vector rotation is not apparent as can be seen from the $\phi$ angle in Figure 3. Note that the plasma beta remains greater than unity because of the unusually high density near $5 / \mathrm{cc}$.

[18] To further investigate the sense of rotation of the velocity vector, we use the technique of Stenuit et al. [2002] and Fujimoto et al. [2003]. With this technique the parameter "a" in Figure $9 \mathrm{f}$ is determined by adding one unit for every rotation of the perturbation vector in the clockwise direction and subtracting one unit for a rotation in the counterclockwise direction. Velocity component $\mathrm{V}_{\mathrm{x}}$ and $\mathrm{V}_{\mathrm{y}}$ are shown in blue in Figure 9b and 9c along with a 10 min running average of these values in green. The deviations from the running average are shown in Figure $9 \mathrm{~d}$ and the total magnitude of the perturbation vector in 9e. Figure 9 clearly shows that clockwise rotations are prevalent up until 0620 and counterclockwise rotations are prevalent after this time. The counterclockwise rotations are easily seen in the $\phi$ angle in Figures 7 and 8 when there is little average $V_{x}$ to subtract. The earlier clockwise rotations occur during the recurring presence of rapidly tailward flowing magnetosheath plasma. These apparent rotations are probably not an indication of bulk rotation of the plasma but are probably due to the negatively increasing $\mathrm{V}_{\mathrm{y}}$ moving the boundary inward such that the spacecraft observes the tailward flowing (negatively increasing) $\mathrm{V}_{\mathrm{x}}$ which creates a clockwise rotating vector.

\section{Simulations}

[19] In an effort to place the Geotail observations in a global context, we employed the global MHD code BATSR-US developed at Center for Space Environment Modeling (CSEM) at the University of Michigan. BATS-R-US is the Global Magnetosphere (GM) module of the Space Weather Modeling Framework (SWMF) designed to model the space environment for various space physics applications [Tóth et al., 2005]. The SWMF contains nine modules that cover the various regions between the Sun and Earth. Simulations presented in this paper utilize only GM and Ionosphere Electrodynamics (IE) modules. In-depth discussions of the simulation scheme used by BATS-R-US can be found in the work of Powell et al. [1999] and Gombosi et al. [2002]. BATSR-US implements a block-based domain-decomposition technique. The simulation grid is composed of many self-similar three-dimensional rectangular blocks arranged in varying degrees of spatial refinement. This adaptive block-based hierarchical data structure permits increases in resolution where and when needed. Our simulation box size in GSM coordinates is $-351 \mathrm{R}_{\mathrm{E}}<\mathrm{X}<33 \mathrm{R}_{\mathrm{E}},|\mathrm{Y}|<96 \mathrm{R}_{\mathrm{E}}$, $|z|<96 R_{E}$, although our interest is in a much smaller region on the dust flank.

[20] The near-Earth boundary of the GM domain is located 2.5 $\mathrm{R}_{\mathrm{E}}$ from the center of the Earth. Near-Earth velocity boundary conditions are determined by the IE module which is a two-dimensional electrostatic potential solver that obtains the field-aligned currents from GM and delivers convection velocity at the boundary back to GM.The height-integrated conductance model used by IE includes effects of EUV, starlight, and particle precipitation [Ridley et al., 2004].

[21] Wind measurements of the IMF and solar wind were projected to the inflow boundary at $\mathrm{X}=33 \mathrm{R}_{\mathrm{E}}$ and used as a boundary condition. The simulations were first initialized with a lengthy run using solar wind parameters and the Earth's dipole tilt at the time 0430 UT on 1 August 1998 (except that $\mathrm{B}_{\mathrm{x}}$ was set to the average value during the time interval of interest 0500-0700 UT). Beginning at 0430, all solar wind parameters were updated with time except that $\mathrm{B}_{\mathrm{x}}$ was kept constant to maintain $\operatorname{div} \mathbf{B}=0$ at the boundary. To further reduce the effects of the initial setup, the code was run for $30 \mathrm{~min}$ (from 0430 to $0500 \mathrm{UT}$ ) prior the meaningful duration of the run, 0500-0700 UT.

[22] The basic grid with about $5 \times 10^{6}$ grid cells at the beginning of the simulation resolves a significant portion of the magnetosphere sunward of $-39 R_{E}$ to $1 / 4 R_{E}$. Fifteen minutes prior to $0500 \mathrm{UT}$, two additional levels of refinement (up to $1 / 16 \mathrm{R}_{\mathrm{E}}$ ) were added using an additional $10^{7}$ grid cells, chosen to give increased resolution in a limited region around the velocity shear layer at the duskside magnetopause. Figure 10 shows two equatorial plane views 


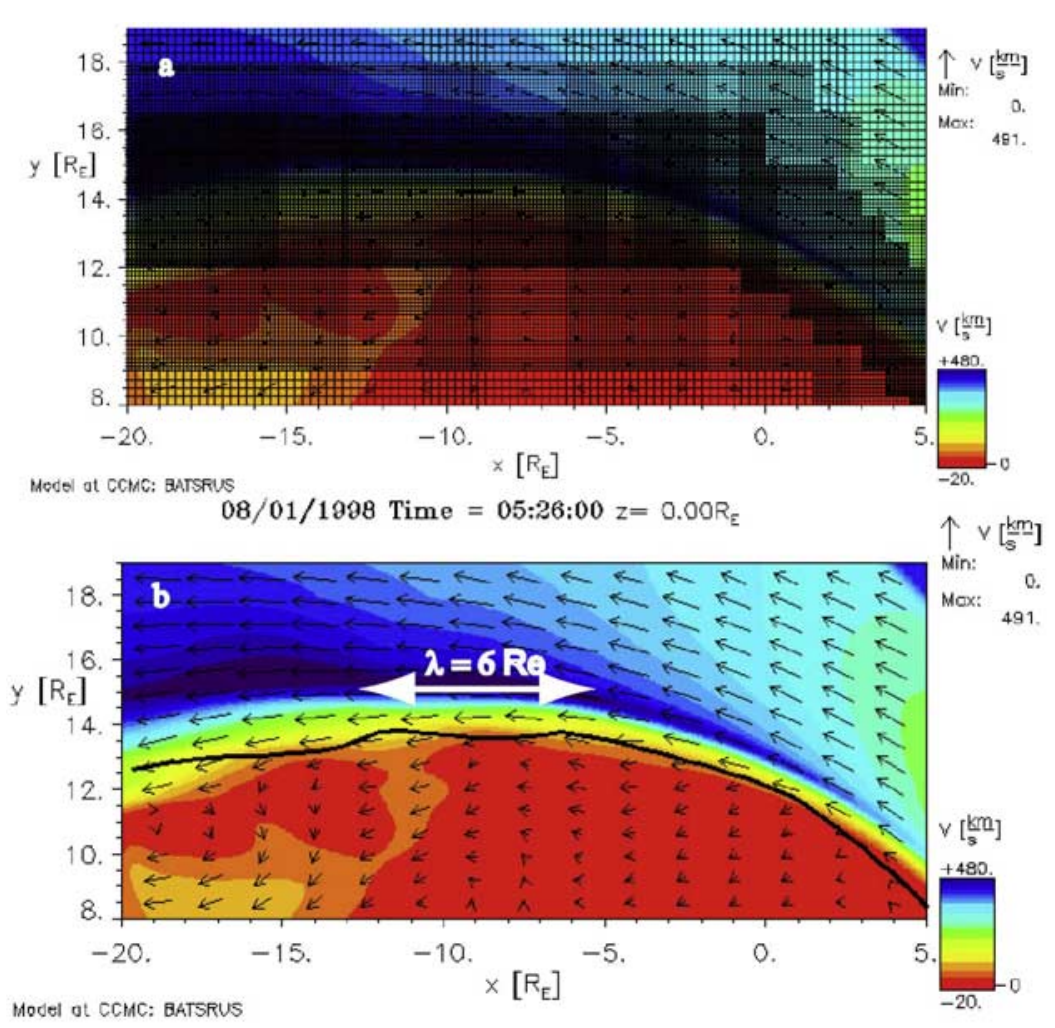

Figure 10. $(a, b)$ Equatorial views of the simulation velocity as shown by both the color coding and the vectors. The two figures are the same except the simulation grid is superimposed on Figure 10a. Waves can be seen on the color boundaries with a wavelength of $\sim 6$ Re. The black line in Figure 10b indicates the last closed field line boundary.

of this limited region of the magnetosphere with the simulation grid superimposed on the top. Maximum resolution in the dark region is $1 / 16 \mathrm{R}_{\mathrm{E}}$ or $400 \mathrm{~km}$. The highly resolved region extends from $-6 \mathrm{Re}$ to $+6 \mathrm{Re}$ in $\mathrm{Z}$ direction.

[23] The figure shows plasma velocity represented by both vectors and color-coding with low velocities in the magnetosphere appearing as red. Waves can be seen as undulations on the color boundaries and the apparent wavelength of $\sim 6 \mathrm{R}_{\mathrm{E}}$ is noted. This wavelength is similar to that deduced from Geotail data on this day obtained by multiplying an observed 3 min period by a representative $200 \mathrm{~km} / \mathrm{s}$ velocity. The black line representing the outer boundary of closed magnetic field lines also shows the undulations.

[24] Another prominent feature in Figure 10 is the peak in magnetosheath velocity just outside the decreasing velocity gradient (shear layer) which itself is almost $1 R_{E}$ thick. Beginning slightly tailward of the dusk terminator, the high magnetosheath velocities are even faster than the solar wind. Examination of other parameters shows that the high velocities are associated with a minima in density and a peak value in B, extremes which are characteristics of the plasma depletion layer [Zwan and Wolf, 1976; Southwood and Kivelson, 1995]. The high-velocity region in Figure 10 is clearly in the magnetosheath as it lies just outside the peak current region (not shown) associated with the small shear in $\mathbf{B}$. This shear is itself near the outer edge of the velocity gradient, which is more than $1 R_{E}$ outside the closed field line boundary. Phan et al. [1994] noted such an increase in velocity in the dayside equatorial region between 0800 and1600 LT but only for cases of low shear in B (i.e., generally northwards fields as in the present case). Such high velocities near the flank magnetopause have also been reported by Petrinec et al. [1997]; Chen et al. [1993] and Phan et al. [1997], again for cases when B is orthogonal to V. Chen et al. [1993] also performed a global simulation and also found enhanced velocities that were faster than the solar wind; however, their high-velocity regions are wider than the current simulation, probably because of their much lower $1 R_{E}$ grid resolution. Their explanations for such enhanced velocities was that equatorial plasma that passed near the subsolar stagnation region is trying to catch up with the interplanetary ends of their field lines that have moved downstream and created a curved field line whose tension accelerates the plasma.

[25] It should be noted that although the simulation produces waves of the right wavelength, it does not produce the complex vortices observed by Geotail. Apparently, the growth rate in the simulation does not permit growth beyond the linear state before the waves move down the tail. We attribute this low growth rate to numerical diffusion in the simulation code that does not allow a thin enough boundary that would lead to a higher growth rate.

[26] To support this contention, we estimate the theoretical KH growth rate expected in the simulation using linear theory [e.g., Walker, 1981; Miura and Pritchett, 1982]. It was determined from the simulation data at 0500 UT that 


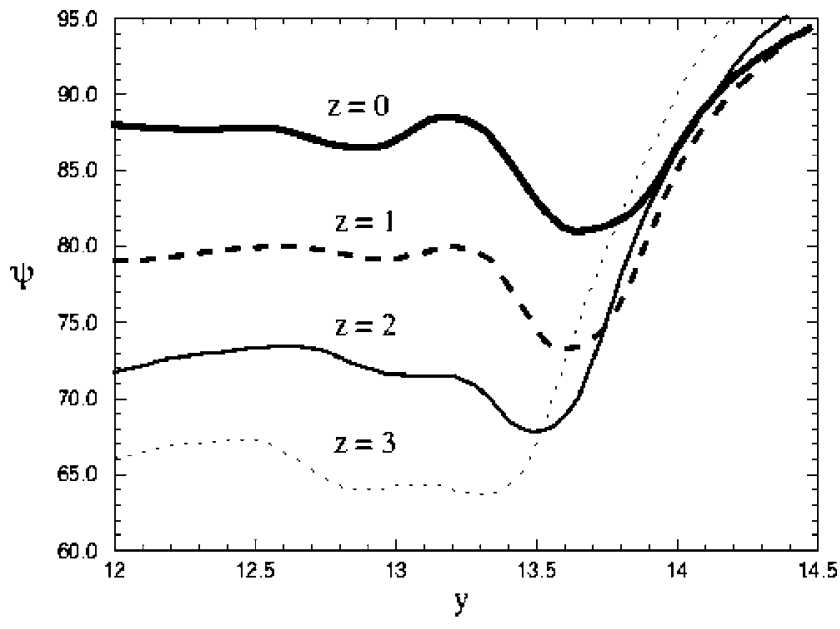

Figure 11. The angle $\psi$ between $\mathbf{k}$ and $\mathbf{B}$ is shown as a function of the perpendicular distance through the boundary layer for different $\mathrm{Z}$ positions. The $\psi$ values less than $90^{\circ}$ will inhibit $\mathrm{KH}$ growth.

the downtail velocity across the boundary layer could be closely approximated as $\left.\mathrm{V}^{\prime}=\mathrm{V}_{0} \tanh \left(\mathrm{y}-\mathrm{y}_{\mathrm{o}}\right) / \mathrm{d}\right)+\mathrm{V}_{1}$ where $\mathrm{y}$ is the distance perpendicular to the boundary, $\mathrm{y}_{0}=13.7$ $\mathrm{R}_{\mathrm{E}}, \mathrm{V}_{0}=230 \mathrm{~km} / \mathrm{s}, \mathrm{V}_{1}=250 \mathrm{~km} / \mathrm{s}$ and $\mathrm{d}=2800 \mathrm{~km}$. We assume the wave vector $\mathbf{k}$ for the most unstable wave is parallel to $\mathrm{V}$ at the velocity peak. Using the $6 \mathrm{R}_{\mathrm{E}}$ wavelength from the simulation, $\mathrm{kd}=0.46$. According to Figure $2 \mathrm{a}$ in the work of Walker [1981], this value would correspond to a normalized growth, $\gamma \mathrm{d} / \mathrm{Vo}$, of approximately 0.2 for an idealized case where the angle $\psi$ between $\mathbf{B}$ and $\mathbf{k}$ is $90^{\circ}$. We then determine $\psi$ at positions along a line through the simulated dusk boundary layer near $X=-3 R_{E}$ and at $Z$ positions $0,1,2$, and $3 \mathrm{R}_{\mathrm{E}}$ as shown in Figure 11 . We find that $\psi$ is less than $90^{\circ}$ both because of the curved dipole-like fields in the boundary layer and also due to further $\mathbf{B}$ distortions in the velocity gradient region where $\psi$ achieves its minimum value. To further compare with the two-diminsional theory of Walker [1981], we choose an average value $\psi=77^{\circ}$ from Figure 11, which is appropriate for a region $\mathrm{Z}= \pm 2 \mathrm{R}_{\mathrm{E}}$, a reasonable approximation given the $6 \mathrm{R}_{\mathrm{E}}$ wavelength. For $\psi=77^{\circ}$, Figure $4 \mathrm{~b}$ of Walker shows that the normalized growth rate is 0.1 , a factor of 2 lower than the $90^{\circ}$ case. This value corresponds to an e-folding time of $12.7 \mathrm{~min}$. In such a time the wave would travel about $30 \mathrm{R}_{\mathrm{E}}$, giving time for wave development but not time for the wave to reach a nonlinear state. The Geotail measurements, in contrast, suggest a smaller d, which would lead to a larger growth rate that could become the nonlinear in the region of observation.

[27] Further observational evidence for the enhance flow velocity layer comes from Figure 12, which displays Geotail CPI and LEP data along with the solar wind data in green for times preceding the 0530 UT magnetopause crossing. Intervals shaded yellow indicate regions with enhanced velocity which we associate with the high-velocity region adjacent to the magnetopause in the simulation. A low solar wind pressure $0400-0420$ UT is caused by a low solar wind density and this low pressure apparently moved the magnetopause out near Geotail. Note that in the extended yellow region adjacent to the magnetopause (dashed line) there are successive velocity peaks that occur with the same period seen later in the boundary layer. This periodicity suggests either in and out motion of the layer or wave-like protrusions into the magnetosheath on the boundary of the layer. The interval 0515-0530 UT is expanded in Figure 13 where a LEP electron energy spectrum is shown in Figure 13a along with the high-resolution magnetic field and ion plasma parameters. Clearly, hot electrons are associated with the high tailward velocity peaks that are associated with hot ions and perturbed magnetic field. (In the highdensity magnetosheath that adjoins the hot electrons, the electron temperature is so low that the high magnetosheath electron fluxes are virtually off scale at the lowest energies in Figure 13.) The enhanced velocity regions in Figure 13 are slightly broader than the hot plasma regions. Similarly, in the earlier two high-velocity yellow regions shown in Figure 12, there are three brief hot-electron, peak-velocity intervals (not shown) within these yellow regions. These observations all suggest that there was an enhanced-velocity magnetosheath region adjacent to the boundary layer but also the outer portion of the boundary layer with an additionally enhanced velocity was briefly observed when the entire structure moved out far enough. This hot electron region may be that found in the simulations of Nakamura et al. [2004] and Takagi et al. [2006] where the high-temperature outer portion of a rolled up vortex has tailward velocity enhanced over that of the adjacent magnetosheath.

[28] By looking at consecutive 1 min simulation plots like Figure 10b, it is possible to track individual wave structures as they move tailward in the boundary layer. The movement of 17 such structures is shown in Figure 14 where their distance down the tail is plotted versus time. The slope of the individual lines is the phase velocity of the waves and

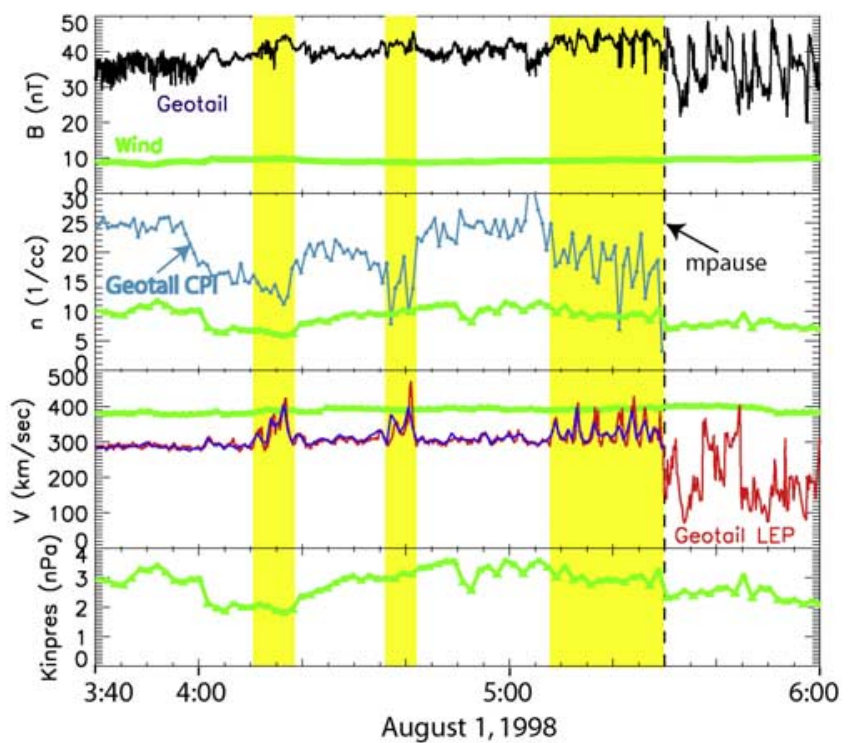

Figure 12. Geotail magnetosheath data along with solar wind data in green for the interval preceding the magnetopause crossing. Enhanced velocities that sometimes exceed the solar wind velocity are seen, when a highvelocity region near the magnetopause moves outward over the spacecraft. 


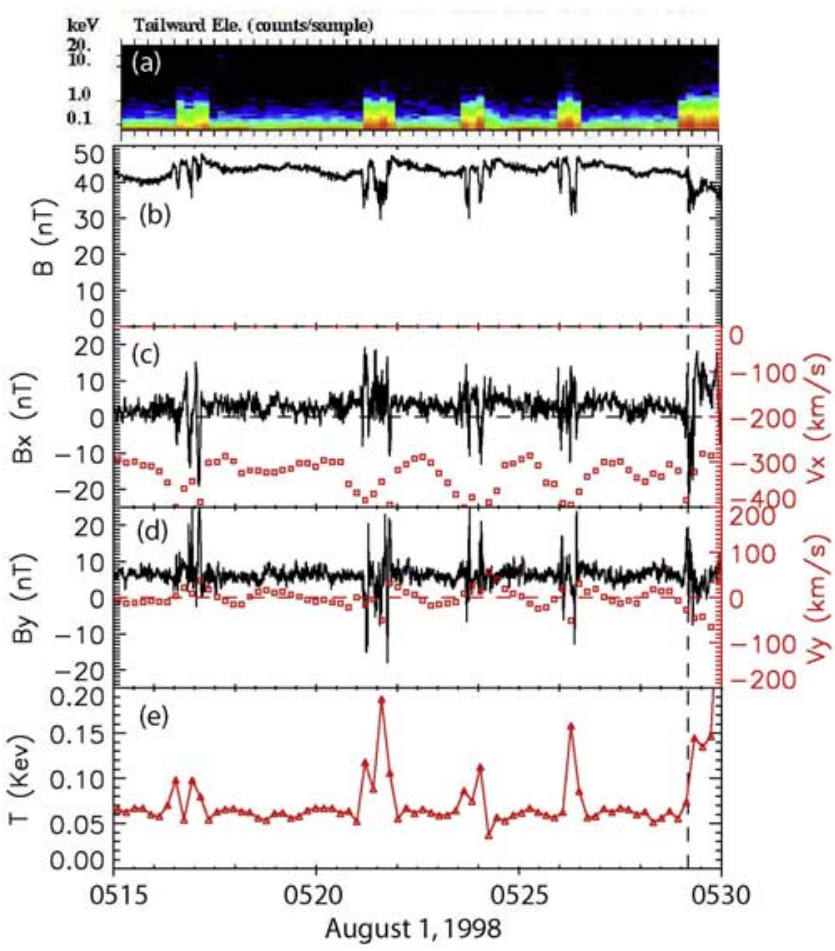

Figure 13. Geotail data showing (a) a LEP electron energy spectrogram, (b) high-resolution magnetic field magnitude, (c,d) $\mathrm{X}$ and $\mathrm{Y}$ components of the magnetic field and velocity, and (e) ion temperature. The high-velocity, hot electrons and protons may be the outer portion of a rolled up vortex as found in simulations of Nakamura et al. [2004] and Takagi et al. [2006].

their average is $223 \pm 36 \mathrm{~km} / \mathrm{s}$. Solar wind pressure shown in the top trace sometimes shows a similar periodicity to the waves (e.g., 0500-0530) suggesting the solar wind variations might be a cause of the waves. At other times, however (e.g., 0550-0605), this is not the case, implying that the $\mathrm{KH}$ instability is operating. Perhaps solar wind perturbations are useful in instigating the $\mathrm{KH}$ instability.

[29] Figure 15 shows simulation data as a function of time at the three locations $\mathrm{X}=-14, \mathrm{Y}=12$ and $\mathrm{Z}=2,0$ and $-2 R_{E}$ as indicated by three colors. Figure 15 a shows the solar wind pressure shifted by $26 \mathrm{~min}$. Increases and decreases in the pressure at 0616 and 0655 can be seen to control the average magnetic field strength in Figure 15b. Waves with periods of $\sim 5$ min are apparent in $\mathrm{B}$ as well as the density in Figure $15 c$ and $B_{x}$ and $B_{y}$ in Figures $15 d$ and $15 \mathrm{e}$. The signs of the $\mathrm{B}_{\mathrm{x}}$ and $\mathrm{B}_{\mathrm{y}}$ components are opposite in the two hemispheres as expected from a dipole-like field. The components at $\mathrm{Z}=0$ are similar to the southern hemisphere, which implies that at this location the sunward tilt of the dipole equatorial plane is more important than the southward curving of the current sheet discussed earlier. Waves increase the strength of the $\mathrm{B}_{\mathrm{x}}$ and $\mathrm{B}_{\mathrm{y}}$ components simultaneously in the two hemispheres and these changes are in phase with $\mathrm{n}$ and $\mathrm{B}_{\mathrm{z}}$ (not shown but essentially equivalent to $\mathrm{B}$ ), all suggesting a compressional component to the waves. Figures $15 \mathrm{f}$ and $15 \mathrm{~g}$ show the $\mathrm{V}_{\mathrm{x}}$ and $\mathrm{V}_{\mathrm{y}}$ components of the velocity relative to a 13 min running average. Figure $15 \mathrm{~h}$ repeats the $\mathrm{V}_{\mathrm{x}}$ and $\mathrm{V}_{\mathrm{y}}$ components from the previous panels in order to show that $\mathrm{V}_{\mathrm{x}}$ tends to lead $\mathrm{V}_{\mathrm{y}}$, as occurs for counterclockwise polarization. This polarization is more clear for smaller Y values away from the boundary (not shown).

\section{Summary and Discussion}

[30] On 1 August 1998 the Geotail spacecraft moved inward through the dusk low-latitude boundary layer during a long interval of almost directly northward IMF. Geotail detected boundary layer waves of three minute period similar to those reported in the past and interpreted as Kelvin-Helmholtz waves. On each wave cycle a region of large-amplitude magnetic fluctuations was seen on exiting a cool dense magnetosheath-like plasma region but on returning to such plasmas the fluctuations were not seen. Such observations seem to rule out a uniform undulating boundary and better support a more complex vortex structure where the fluctuations occur within the vortex. Associated with this structure are mixed magnetosheath and magnetosphere plasmas and velocity perturbations that are consistent with both in and out motions and vortical rotations. Total pressure minima are likely associated with the center of the vortices.

[31] As Geotail moved further inward magnetosheath-like plasmas and fluctuating fields were detected less often, but periodic velocity waves continued that exhibited counterclockwise polarization. Such waves just inside the boundary layer have not often been studied in the past but they are likely related to magnetotail waves detected by ISEE $1 / 2$ and termed magnetotail vortices [Hones et al., 1978, 1981, 1983; Birn et al., 1985]. These ISEE measurements revealed rotating $\mathrm{B}$ and $\mathrm{V}$ vectors with periods of $10 \pm 5 \mathrm{~min}$ although they never persisted beyond five rotations. They were observed throughout the tail but less frequently near midnight. The two ISEE spacecraft were used to determine wavelengths of 5-30 $\mathrm{R}_{\mathrm{E}}$. The observed ISEE vectors rotated clockwise in the dawn hemisphere and counter clockwise in the dusk hemisphere and hence were similar to the counterclockwise sense of rotation seen by Geotail at dusk. These ISEE waves were thought to be caused by KH instability of the magnetopause although no direct evidence

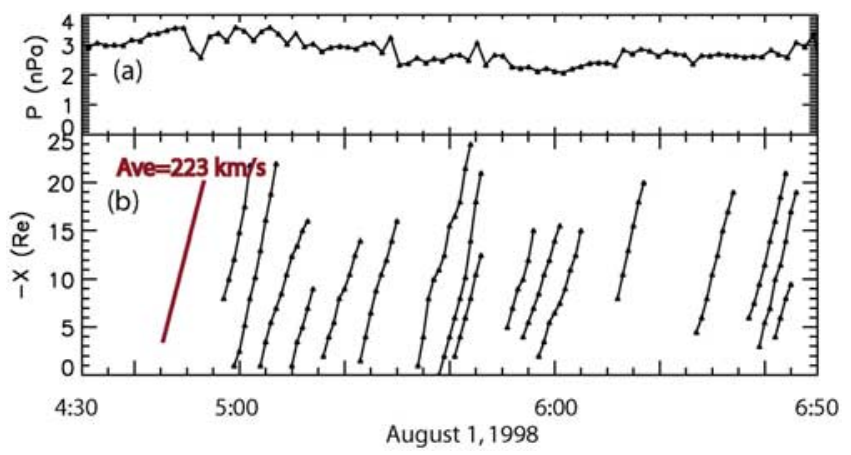

Figure 14. $(a, b)$ Downtail motion of individual structures in the simulation leads to the plot of distance down the tail versus time in Figure 14b. The solar wind kinetic pressure input to the simulation is in Figure 14a. The average phase velocity of the waves in the spacecraft frame is similar to plasma velocity near the boundary. 


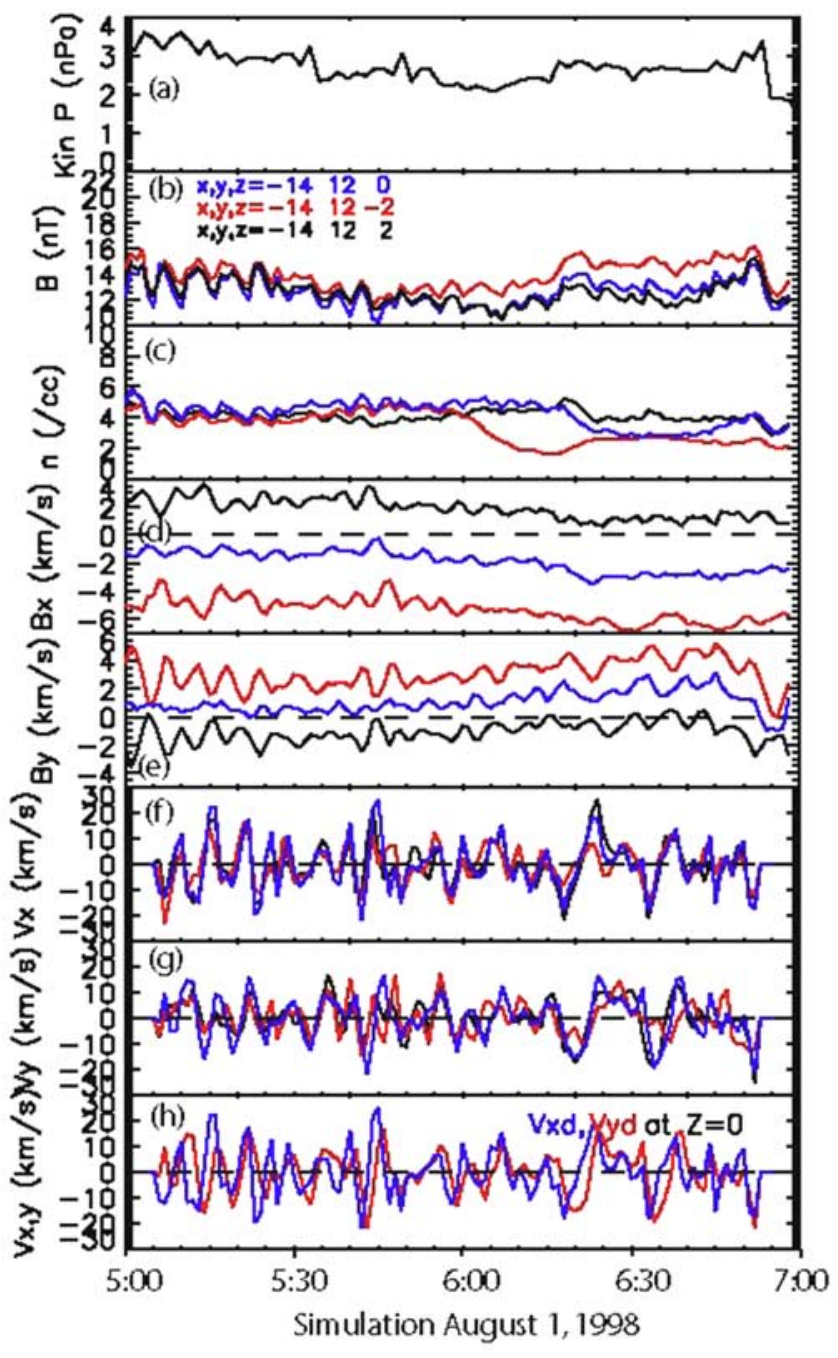

Figure 15. Data from three magnetosphere positions, $X=$ $-14, Y=12$, and $Z=0$ and \pm 2 , showing (a) solar wind kinetic pressure, (b) magnetic field strength, (c) density, ( $d$ and $e$ ) the $B_{x}$ and $B_{y}$ magnetic field components, ( $f$ and $g$ ) simulation velocities relative to a 13 min running average to show the similarities at the three positions, and (h) $V_{x}$ and $\mathrm{V}_{\mathrm{y}}$ components at $\mathrm{Z}=0$ to indicate the phase differences indicating counterclockwise polarization.

was presented. Although the Geotail waves are on the low end of the ISEE range of periods, Geotail spectra (not shown) show that the periods increased gradually from $3 \mathrm{~min}$ to $5 \mathrm{~min}$ between 0530 and 0700 . There is also evidence that $\mathrm{KH}$ boundary waves have longer periods further down the tail. The fact that ISEE never saw more than five consecutive rotations in contrast to the ongoing waves observed by Geotail may be due to the unusual persistence of the very northward IMF on 1 August 1998. ISEE did not detect any relation of the waves to geomagnetic activity (north versus south IMF) but this may simply mean the $\mathrm{KH}$ waves are more common than is generally appreciated. All things considered, it seems possible that as Geotail moved inward from the boundary layer, it saw the evolution of $\mathrm{KH}$ boundary layer waves to the reported tail vortices.
[32] The MHD simulation provides a context for interpreting the Geotail observations, but it is important to compare and contrast the two approaches. We should first explain that we do not make direct comparisons between simulation and observation because at the time Geotail passes through the boundary layer this satellite location is just outside the simulated magnetosphere. Given more computer time, the simulation could have been rerun with a lower solar wind pressure and the spatial agreement improved, but this is not practical and does not seem necessary given the fact that the solar wind conditions do not change importantly over the interval.

[33] In several ways the simulation agreed with observations. The simulation clearly revealed a wavelength of $\sim 6 \mathrm{R}_{\mathrm{E}}$ that agreed quite well with that deduced from observations. A region of enhanced magnetosheath velocities in the simulation was apparent adjacent to the magnetosheath that explained observations and will help set boundary conditions for future local simulations. Such enhanced velocities will in general promote $\mathrm{KH}$ instability but a distortion of $\mathbf{B}$ in the shear region of the simulation can be expected to impede KH growth. Further brief Geotail velocity enhancements above the magnetosheath enhancements were associated with hot plasma and fluctuating fields and may be the features of rolled up vortices as seen in the local simulations of Nakamura et al. [2004] and Takagi et al. [2006]. Magnetosphere waves in our global simulation exhibited counterclockwise polarization that agreed with observations.

[34] When considering differences between observations and simulations, it should be appreciated that MHD cannot be expected to reproduce the fine-scale structure seen in the data, no matter what the spatial resolution in the simulation. Although the reported simulation achieved unusually good $(\sim 400 \mathrm{~km})$ spatial resolution in the boundary region, this still-limited resolution along with diffusion in the simulation code together probably prevented representation of the actual large velocity gradients. This fact is thought to have led to a less-than-realistic growth rate in the simulation and waves that did not develop beyond the linear stage in the region of interest. The magnetosphere waves in the simulation near the Geotail observation point had slightly longer periods and they were more elliptically polarized than the observations. On the other hand, at a more tailward location the simulation waves were more circular. With a higher growth rate, perhaps such circular polarization might have occurred further upstream where it was observed.

[35] There is also some correspondence between waves in the solar wind pressure and those observed in the simulation, raising the question of whether the simulation might simply be responding to the solar wind pressure changes as proposed by Kepko and Spence [2002, 2003]. The largestamplitude solar wind waves from 0450 to 0510 in Figure 14 indeed correspond quite well to the observed waves, but at later times waves are observed with little evidence for solar wind waves. Similarly, in Figure 15 there are few solar wind waves while simulation waves are occurring. It also seems unlikely that solar wind compressions created the waves observed by Geotail. The clearest solar wind waves from 0455 to 0510 occurred while Geotail was still in the magnetosheath and subsequently there were few solar wind waves. Although solar wind waves were generally not 
considered in the several boundary layer wave examples in the literature and cited earlier, the northward IMF conditions that are apt to lead to $\mathrm{KH}$ instabilities seem to be common to all events. It is, however, possible that solar wind pressure changes might be a factor in triggering the $\mathrm{KH}$ instability.

\section{Conclusions}

[36] Waves on the dusk low-latitude boundary layer were observed by the Geotail spacecraft on 1 August 1998 during an extended period of northward IMF. These observations lend further support to the idea that the boundary is KelvinHelmholtz unstable under such conditions. This conclusion is supported by the observation of a region of large rapid magnetic field fluctuations up to $50 \mathrm{nT} / \mathrm{s}$ that are likely twisted fields within a vortex that leads to reconnection that allow plasma to enter the magnetosphere. As Geotail moved through the LLBL into the magnetosphere proper, it continued to detect velocity waves that were circularly polarized in a counterclockwise sense. Such waves are probably the "magnetotail vortices" studied earlier by the ISEE spacecraft [Hones et al., 1978, 1981, 1983; Birn et al., 1985] and proposed earlier as being due to the $\mathrm{KH}$ instability. A global MHD simulation of this event exhibited characteristics seen in the data. Waves were seen in the simulation although they probably had not reached as nonlinear a stage as in observations. The simulation waves had counterclockwise polarization similar to observations although they were more elliptical than the observations. The simulation revealed a region of enhanced magnetosheath flow velocity adjacent to the magnetopause that was also noted in the observations. This high-velocity flow was associated with lower plasma density and increased B and hence can be identified as a plasma depletion layer. This enhancement in velocity should promote the $\mathrm{KH}$ instability and make it occur more frequently under northward IMF conditions. Additional brief Geotail velocity enhancements above the magnetosheath enhancements were associated with hot plasma and fluctuating fields and may be rolled up vortices seen in the local simulations of Nakamura et al. [2004] and Takagi et al. [2006].

[37] Acknowledgments. We gratefully acknowledge A. J. Lazarus and R. P Lepping for providing Wind plasma and magnetic field data and L. A. Frank and W. R. Patterson for providing Geotail CPI data.

[38] Amitava Bhattacharjee thanks Ian Mann and another reviewer for their assistance in evaluating this paper.

\section{References}

Birn, J., E. W. Hones Jr., S. J. Bame, and C. T. Russell (1985), Analysis of 16 plasma vortex events in the geomagnetic tail, J. Geophys. Res., 90, 7449.

Chen, S.-H., and M. G. Kivelson (1993), On nonsinusoidal waves at the Earth's magnetopause, Geophys. Res. Lett., 20, 2699.

Chen, S.-H., M. G. Kivelson, J. T. Gosling, R. J. Walker, and A. J. Lazarus (1993), Anomalous aspects of magnetosheath flow and of the shape and oscillations of the magnetopause during an interval of strongly northward interplanetary magnetic field, J. Geophys. Res., 98, 5727.

Fairfield, D. H., A. Otto, T. Mukai, S. Kokubun, R. P. Lepping, J. T. Steinberg, A. J. Lazarus, and T. Yamamoto (2000), Geotail observations of the Kelvin-Helmholtz instability at the equatorial magnetotail boundary for parallel northward fields, J. Geophys. Res., 105, 21,159.

Fairfield, D. H., C. J. Farrugia, T. Mukai, T. Nagai, and A. Federov (2003), Motion of the dusk flank boundary layer caused by solar wind pressure changes and the Kelvin-Helmholtz instability: 10-11 January 1997, J. Geophys. Res., 108(A12), 1460, doi:10.1029/2003JA010134.
Farrugia, C. J., et al. (2000), Coordinated Wind, Interball/tail, and ground observations of Kelvin-Helmholtz waves at the near-tail, equatorial magnetopause at dusk: January 11, 1997, J. Geophys. Res., 105, 7639.

Frank, L. A., K. L. Ackerson, W. R. Paterson, J. A. Lee, M. R. English, and G. L. Pickett (1994), The Comprehensive Plasma Instrumentation (CPI) for the Geotail spacecraft, J. Geomagn. Geoelectr., 46, 23.

Fujimoto, M., and T. Terasawa (1994), Anomalous ion mixing within an MHD scale Kelvin-Helmholtz vortex, J. Geophys. Res., 99, 8601.

Fujimoto, M., and T. Terasawa (1995), Anomalous ion mixing within an MHD scale Kelvin-Helmholtz vortex: 2. Effects of inhomogeneity, J. Geophys. Res., 100, 12,025.

Fujimoto, M., T. Tonooka, and T. Mukai (2003), Vortex-like fluctuations in the magnetotail flanks and their possible roles in plasma transport, in Earth's Low-Latitude Boundary Layer, Geophys. Monogr. Ser, vol. 133, edited by P. T. Newell and T. Onsager, p. 241, AGU, Washington, D. C.

Gombosi, T. I., G. Toth, D. L. DeZeeuw, K. G. Hansen, K. Kabin, and K. G. Powell (2002), Semi-relativistic magnetohydrodynamics and physicsbased convergence acceleration, J. Comput. Phys., 177, 176.

Hasegawa, H., M. Fujimoto, T.-D. Phan, H. Reme, A. Balogh, M. W. Dunlop, C. Hashimoto, and R. TanDokoro (2004), Transport of solar wind into Earth's magnetosphere through rolled-up Kelvin-Helmholtz vortices, Nature, 430, 755.

Hones, E. W., Jr., G. Paschmann, S. J. Bame, J. R. Asbridge, N. Sckopke, and K. Schindler (1978), Vortices in magnetospheric plasma flow, Geophys. Res. Lett., 5, 1059.

Hones, E. W., Jr., J. Birn, S. J. Bame, J. R. Asbridge, G. Paschmann, N. Sckopke, and G. Haerendel (1981), Further determination of the characteristics of magnetospheric plasma vortices with ISEE 1 and 2, J. Geophys. Res., 86, 814.

Hones, E. W., Jr., J. Birn, S. J. Bame, and C. T. Russell (1983), New observations of plasma vortices and insights into their interpretation, Geophys. Res. Lett., 10, 674.

Keller, K. A., and R. L. Lysak (1999), A two-dimensional simulation of Kelvin-Helmholtz instability with magnetic shear, J. Geophys. Res., 104, 25,097 .

Kepko, L., and H. E. Spence (2002), ULF waves in the solar wind as direct drivers of magnetospheric pulsations, Geophys. Res. Lett., 29(8), 1197, doi:10.1029/2001GL014405.

Kepko, L., and H. E. Spence (2003), Observations of discrete, global magnetospheric oscillations directly driven by solar wind density variations, J. Geophys. Res., 108(A6), 1257, doi:10.1029/2002JA009676.

Kivelson, M. G., and S.-H. Chen (1995), The Magnetopause: Surface waves and instabilities and their possible dynamical consequences, Physics of the Magnetopause, Geophys. Monogr. Ser., vol. 90, edited by P. Song, B. U. O. Sonnerup, and M. F. Thomsen, pp. 257-268, AGU, Washington, D. C

Knoll, D. A., and J. U. Brackbill (2002), The Kelvin-Helmholtz instability, differential rotation, and three-dimensional, localized, magnetic reconnection, Phys. Plasmas, 9, 3775, doi:10.1063/1.1494070.

Kokubun, S., H. Kawano, M. Nakamura, T. Yamamoto, K. Tsuruda, H. Hayakawa, A. Matsuoka, and L. A. Frank (1994), Quasi-periodic oscillations of the magnetopause during northward sheath magnetic field, Geophys. Res. Lett., 21, 2883.

Lai, S. H., and L. H. Lyu (2006), Nonlinear evolution of the MHD KelvinHelmholtz instability in a compressible plasma, J. Geophys. Res., 111, A01202, doi:10.1029/2004JA010724

Lepping, R. P., et al. (1995), The Wind magnetic field investigation, in The Global Geospace Mission, edited by C. T. Russell, p. 207, Kluwer Acad., Boston, Mass.

Li, X., and M. Temerin (2001), The electron radiation belt, Space Sci. Rev, 95, 569.

Mann, I. R., et al. (2002), Coordinated ground-based and Cluster observations of large amplitude global magnetospheric oscillations during a fast solar wind speed interval, Ann. Geophys., 20, 405.

Manuel, J. R., and J. C. Samson (1993), The spatial development of the low latitude boundary layer, J. Geophys. Res., 98, 17,367.

Matsumoto, Y., and M. Hoshino (2004), Onset of turbulence induced by a Kelvin-Helmholtz vortex, Geophys. Res. Lett., 31, L02807, doi:10.1029/ 2003GL018195.

Matsumoto, Y., and M. Hoshino (2006), Turbulent mixing and transport of collisionless plasmas across a stratified velocity shear layer, J. Geophys. Res., 111, A05213, doi:10.1029/2004JA010988.

Mills, K. J., A. W. Longbottom, A. N. Wright, and M. S. Ruderman (2000), Kelvin-Helmholtz instability on the magnetospheric flanks: An absolute and convective instability approach, J. Geophys. Res., 105, 27,685 .

Miura, A. (1984), Anomalous transport by magnetodynamic KelvinHelmholtz instabilities in the solar wind-magnetosphere interaction, J. Geophys. Res., 89, 801. 
Miura, A. (1987), Simulation of Kelvin-Helmholtz instability at the magnetospheric boundary, J. Geophys. Res., 92, 3195.

Miura, A. (1990), Kelvin-Helmholtz instability for supersonic shear flow at the magnetospheric boundary, Geophys. Res. Lett., 17, 749.

Miura, A. (1995), Kelvin-Helmholtz instability at the magnetopause: Computer simulations, in Physics of the Magnetopause, Geophys. Monogr Ser., vol. 90, edited by P. Song, B. U. O. Sonnerup, and M. F. Thomsen, pp. 285-291, AGU, Washington, D. C.

Miura, A. (2003), Nonideal high- $\beta$ magnetohydrodynamic KelvinHelmholtz instability driven by the shear in the ion diamagnetic drift velocity at the subsolar magnetopause, J. Geophys. Res., 108(A2), 1076, doi:10.1029/2002JA009563.

Miura, A., and P. L. Pritchett (1982), Nonlocal stability analysis of the MHD Kelvin-Helmholtz instability in a compressible plasma, J. Geophys. Res., 87, 7431.

Mukai, T., S. Machida, Y. Saito, M. Hirahara, T. Terasawa, N. Kaya, T. Obara, M. Ejiri, and A. Nishida (1994), The low energy particle (LEP) experiment onboard the GEOTAIL satellite, J. Geomagn. Geoelectr., 46, 669.

Nakamura, T. K. M., and M. Fujimoto (2005), Magnetic reconnection within rolled-up MHD-scale Kelvin-Helmholtz vortices: Two-fluid simulations including finite electron inertial effects, Geophys. Res. Lett., 32, L21102, doi:10.1029/2005GL023362.

Nakamura, T. K. M., D. Hayashi, M. Fujimoto, and I. Shinohara (2004), Decay of MHD-scale Kelvin-Helmholtz vortices mediated by parasitic electron dynamics, Phys. Rev. Lett., 92, 145001.

Nakamura, T. K. M., M. Fujimoto, and A. Otto (2006), Magnetic reconnection induced by weak Kelvin-Helmholtz instability and the formation of the low-latitude boundary layer, Geophys. Res. Lett., 33, L14106, doi:10.1029/2006GL026318.

Nykyri, K., and A. Otto (2001), Plasma transport at the magnetosphereic boundary due to reconnection in Kelvin-Helmholtz vortices, Geophys. Res. Lett., 28, 3565.

O'Brien, T. P., and R. L. McPherron (2003), An empirical dynamic equation for energetic electrons at geosynchronous orbit, J. Geophys. Res. 108(A3), 1137, doi:10.1029/2002JA009324.

Ogilvie, K. W., and R. J. Fitzenreiter (1989), The Kelvin-Helnholtz instability at the magnetopause and inner boundary layer surface, J. Geophys. Res., 94, 15,113.

Ogilvie, K. W., et al. (1995), SWE, a comprehensive plasma instrument for the Wind spacecraft, in The Global Geospace Mission, edited by C. T. Russell, p. 55, Kluwer Acad., Boston, Mass.

Otto, A., and D. H. Fairfield (2000), Kelvin-Helmholtz instability at the magnetotail boundary: MHD simulation and comparison with Geotail observations, J. Geophys. Res., 105, 21,175.

Owen, C. J., G. G. T. M.Taylor, I. C. Krauklis, A. N. Fazakerley, M. W. Dunlop, and J. M. Bosqued (2004), Cluster observations of surface waves on the dawn flank magnetopause, Ann. Geophys., 22, 971-983.

Phan, T.-D., G. Paschmann, W. Baumjohann, N. Sckopke, and H. Luhr (1994), The magnetosheath region adjacent to the dayside magnetopause: AMPTE/IRM observations, J. Geophys. Res., 99, 121.

Phan, T. D., et al. (1997), Low-latitude dusk flank magnetosheath, magnetopause, and boundary layer for low magnetic shear: Wind observations, J. Geophys. Res., 102, 19,883.

Petrinec, S. P., T. Mukai, A. Nishida, T. Yamamoto, T. K. Nakamura, and S. Kokubun (1997), GEOTAIL observations of magnetosheath flow near the magnetopause, using WIND as a solar wind monitor, J. Geophys. Res., 102, 26,943.

Powell, K. G., P. L. Roe, T. J. Linde, T. I. Gombosi, and D. L. DeZeeuw (1999), A solution-adaptive upwind scheme for ideal magnetohydrodynamics, J. Comput. Phys., 154, 284.
Ridley, A. J., T. I. Gombosi, and D. L. De Zeeuw (2004), Ionospheric control of the magnetosphere: conductance, Ann. Geophys., 22, 567.

Seon, J., L. A. Frank, A. J. Lazarus, and R. P. Lepping (1995), Surface waves on the tailward flanks of the Earth's magnetopause, J. Geophys. Res., 100, 11,907.

Shue, J.-H., et al. (1998), Magnetopause location under extreme solar wind conditions, J. Geophys. Res., 103, 17,691.

Slinker, S. P., J. A. Fedder, D. G. Sibeck, J. G. Lyon, L. A. Frank, and T. Mukai (2003), Simulation of magnetopause oscillations observed January 9, 1996, Geophys. Res. Lett., 30(11), 1569, doi:10.1029/ 2003GL017063.

Southwood, D. J., and M. G. Kivelson (1995), Magnetosheath flow near the subsolar magnetopause: Zwan-Wolf and Southwood-Kivelson theories reconciled, Geophys. Res. Lett., 22, 3275.

Stenuit, H., et al. (2002), Multispacecraft study on the dynamics of the dusk-flank magnetosphere under northward IMF: 10-11 January 1997 J. Geophys. Res., 107(A10), 1333, doi:10.1029/2002JA009246.

Takagi, K., C. Hashimoto, H. Hasegawa, M. Fujimoto, and R. TanDokoro (2006), Kelvin-Helmholtz instability in a magnetotail flank-like geometry: Three-dimensional MHD simulations, J. Geophys. Res., 111, A08202, doi:10.1029/2006JA011631.

Takahashi, K., D. G. Sibeck, P. T. Newell, and H. E. Spence (1991), ULF waves in the low latitude boundary layer and their relationship to magnetospheric pulsations: A multisatellite observation, J. Geophys. Res., 96, 9503

Thomas, V. A. (1995), Three-dimensional kinetic simulation of the KelvinHelmholtz instability, J. Geophys. Res., 100, 19,429.

Thomas, V. A., and D. Winske (1993), Kinetic simulations of the KelvinHelmholtz instability of the magnetopause, J. Geophys. Res., 98, 11,425.

Tóth, G., et al. (2005), Space Weather Modeling Framework: A new tool for the space science community, J. Geophys. Res., 110, A12226, doi:10.1029/2005JA011126.

Tsyganenko, N. A., and D. H. Fairfield (2004), Global shape of the magnetotail current sheet as derived from Geotail and Polar data, J. Geophys. Res., 109, A03218, doi:10.1029/2003JA010062.

Walker, A. D. M. (1981), The Kelvin-Helmholtz instability in the lowlatitude boundary layer, Planet. Space Sci., 29, 1119.

Wright, A. N., K. J. Mills, M. S. Ruderman, and L. Brevdo (2000), The absolute and convective instability of the magnetospheric flanks, J. Geophys. Res., 105, 385.

Wright, A. N., K. J. Mills, A. W. Longbottom, and M. S. Ruderman (2002), The nature of convectively unstable waveguide mode disturbances on the magnetospheric flanks, J. Geophys. Res., 107(A9), 1242, doi:10.1029/ 2001JA005091.

Zwan, B. J., and R. A. Wolf (1976), Depletion of solar wind plasma near a planetary boundary, J. Geophys. Res., 81, 1636.

D. H. Fairfield and M. M. Kuznetsova, Laboratory for Solar and Space Physics, NASA Goddard Space Flight Center, Greenbelt, MD 20771, USA. (donald.fairfield@nasa.gov)

T. I. Gombosi and A. J. Ridley, Center for Space Environment Modeling, University of Michigan, 2455 Hayward Street, Ann Arbor, MI 48109-2143, USA.

T. Mukai, Institute of Space and Astronautical Science, Sagamihara, Kanagawa, Japan.

T. Nagai, Earth and Planetary Sciences, Tokyo Institute of Technology, Tokyo, Japan. 